\title{
Wholesomeness and Safety of Meat of Laying Hens Fed Irradiated Aflatoxin $B_{1}$-contaminated Diet
}

\author{
M. Diaa El-Din H. Farag1, E.A. Abdallah², A.M. Abdul Azeem ${ }^{1 *}$ and Nashwa \\ A.H.Ahmed ${ }^{3}$ \\ ${ }^{1}$ Food Irradiation Research Department, National Center for Radiation Research \& \\ Technology, Atomic Energy Authority, Cairo; ' Animal Production Research Institute, \\ Giza, Cairo and ${ }^{3}$ Hormones Unit, Chemistry Department, Animal Health Research \\ Institute, Giza, Cairo, Egypt.
}

\begin{abstract}
$\mathbf{T}$ HE AIM of this study is to evaluate the effect of gamma $(\gamma)$ radiations on the safety of processed diets to eliminate the negative effects of aflatoxin- $\mathrm{B}_{1}\left(\mathrm{AFB}_{1}\right)$ on the health of consumers. One hundred fifty adult female Westar rats were used. The rats were divided into 5 experimental groups $(G) . G_{1}$; rats served as a control which were fed a meal of flesh (breast and thigh muscles) and organs (liver, kidney, spleen and heart) of laying hens that fed before slaughtering diet non-contaminated with $A F B_{1} . G_{2}$; the rats were fed flesh of laying hens fed before slaughtering contaminated diet with $0.2 \mathrm{mg} \mathrm{AFB}_{1} \mathrm{~kg}^{-1}, \mathrm{G}_{3}, \mathrm{G}_{4}$ and $\mathrm{G}_{5}$ rats were fed flesh and organs of slaughtered laying hens fed before being slaughtered contaminated diet with $0.2 \mathrm{mg} \mathrm{AFB}_{1}$ and treated with $\gamma$-irradiation at 10,20 and $30 \mathrm{kGy}$, respectively. The feeding continued for 3 weeks (experimental duration), then followed by another 3 weeks period on commercial non-contaminated ration (recovery duration). The obtained results showed that the feed intake, live body weight, blood total protein, albumin, and globulin were significantly decreased $(P \leq 0.05)$ in rats of $\mathrm{G}_{2}$ during the experimental duration compared to $\mathrm{G}_{1}$. However, an improvement was observed in all parameters for rats of $G_{3}, G_{4}$ and $G_{5}$. This improvement was parallel with increasing the dose levels of $\gamma$-ray (at 10,20 and 30kGy), respectively. On the other hand, $\mathrm{G}_{2}$ showed an increase in the relative organ weights and $\mathrm{AFB}_{1}$ residues in breast tissues and organs including (liver, kidneys, spleen and heart), creatinine, liver function including aspertate aminotransferase (AST), alanine aminotransferase (ALT) and alkaline phosphatase (ALP). The experimental regimes for rats of $G_{3}, G_{4}$ and $G_{5}$ were effective at reducing the deleterious effect of $\mathrm{AFB}_{1}$ on residues in breast tissues and organs. They normalized the relative organ weights as a function of radiation doses. Moreover, an improvement in serum liver enzymes, thyroid hormones and sexual hormones occurred, which reflects their effects on metabolism and reproductive efficiency of rats and this improvement was proportional with increasing the radiation doses up to $30 \mathrm{kGy}$. It could be concluded that the radiation processing (at the applied radiation doses) of contaminated diet with $\mathrm{AFB}_{1}$ has significantly improved all parameters $(\mathrm{P}<0.05)$ which were negatively affected by AFs. Also, improvements were noticed in $\mathrm{G}_{3}$ and $\mathrm{G}_{4}$. A greater amelioration was obtained for group $\mathrm{G}_{5}$ followed a recovery phase.
\end{abstract}

Keywords: Aflatoxins- $\mathrm{B}_{1}$, Rat, Fed, Contaminated diet, Safety, Radiation processing.

\section{Introduction}

Food and feed safety are fundamental requirements to guarantee human health and there is a worldwide solicitude about the dangers of food poisoning (Chibanga et al., 2014). Food and feed are susceptible to be contaminated with mold species belonging to Aspergillus genus, mainly
Aspergillus flavus and Aspergillus parasiticus. These moulds are capable of producing toxic secondary metabolites called aflatoxins $\left(\mathrm{B}_{1}, \mathrm{~B}_{2}\right.$, $\mathrm{G}_{1}$ and $\mathrm{G}_{2}$ ). Among these aflatoxins, aflatoxins $\mathrm{B}_{1}\left(\mathrm{AFB}_{1}\right)$ is the most important member of mycotoxins showing hepatoxic, terategenic, immunosuppressive and potential carcinogenic that pose severe hazards to animal and human 
health. It is classified by the International Agency for Research on Cancer (IARC) as group 1 carcinogen (IARC, 2002).

Acute or chronic aflatoxicosis in poultry results in diminished egg and meat production, liver necrosis and hyperplasia of the bile duct leading to reduced proteins, fatty acids digestibility (Hussein \& Brasel, 2001), and immunosuppressive (Bailey et al., 2006 and Shi et al., 2006). The biological mycotoxins toxicity depends on the type, dose and duration of mycotoxins intake, species, gender, genetic and animal age, general health, immune and nutrition, as well as certain environmental factors (Bryden, 2007). However, when focusing on how AFs play a role in food safety, solicitude should be limited to AFs that are known to be exposed directly by consuming the contaminated commodities or transferred from feed to food of animal origin, as this food represents a significant route of exposure for humans (Bhat et al., 2010).

The permissible level of aflatoxins adopted in most of the world countries is 5ppb as level of human contamination risk (Yosef et al., 2013). AFs residues in food are a major harmful to public health and could potentially create health problems as they suppress the immune response, hepatotoxic and nephrotoxic effects. The problem with AFs does not end with production losses as many of them are readily transferred to the poultry production (egg/meat) (Denli et al., 2009). AF is metabolized in the body into active metabolites and accumulates in egg, muscle tissues, organs of liver, kidney, breast, legs, and gizzard and blood. $\mathrm{AFB}_{1}$ transfers from feed to food and metabolized into milk as $\mathrm{AFM}_{1}$ and $\mathrm{B}_{2}$ in the liver, $\mathrm{AFB}_{1}$ to aflatoxin $B_{1}$ to eggs as aflatoxicol. AFs residues may also appear in body tissues and samples collected from the poultry feed AFs contaminated rations showed detectable levels of AFs in liver and their edible tissues like liver and muscle (Bintvihok et al., 2002).

Since the contamination of food and feed with mycotoxins in particular with $\mathrm{AFB}_{1}$ is unavoidable, it is important to seek for possible way to prevent it, through the inhibition of aflatoxgenic moulds growth, or by reduction of the presence $\mathrm{AFB}_{1}$ in food and feed (Kabak et al., 2006).

Food/feed safety is one of the main challenges for technology. Food/ feed irradiation is one among many of available technologies that has been recognized as a reliable and safe method for the preservation of food and feed that contributes to improving the hygienic quality and nutritional value of them (EPA, 2013). Food irradiation is a processing technology that aims at the improvement of food safety and it is a physical method of food processing that involves exposing prepackaged or in bulk foodstuffs to ionizing energy to eliminate the microbial load or to control the presence of fungi and mycotoxins production in food and in feed (Farkas, 1989; Waje et al., 2009 and Markov et al., 2015). The objective of this study is to evaluate and explore the adverse effects of feeding rats on flesh and organs of laying hens fed before slaughtering $\mathrm{AFB}_{1}$ - contaminated diet and irradiated $\mathrm{AFB}_{1}$-contaminated diet as a sensitive animal model to ensure the safety of processed materials through radiation treatments for consumption. In addition, the study aims to investigate the effect of $\mathrm{AFB}_{1}$ on the studied indices through AFs free duration (recovery duration).

\section{Materials and Methods}

The experimental work of the present study was carried out at the Food Irradiation Department, National Center for Radiation Research and Technology, Atomic Energy Authority, Cairo, Egypt, and the Animal Production Research Institute, Ministry of Agriculture, Dokki, Giza and Animal Health Research Institute, Ministry of Agriculture, Giza, Egypt.

\section{Experimental design}

Five groups, of 60 laying hens each, were contributed in this study. The first group was fed daily on non-contaminated $\mathrm{AFB}_{1}$ ration and served as a control. The second group was fed contaminated $\mathrm{AFB}_{1}$ ration $\left(0.2 \mathrm{mg} \mathrm{AFB}_{1} \mathrm{~kg}^{-1}\right)$ according to Farag et al. (2017). The third to fifth groups were fed $\gamma$-irradiated contaminated ration $\left(0.2 \mathrm{mg} \mathrm{AFB}_{1} \mathrm{~kg}^{-1}\right.$ irradiated with 10 , 20 and $30 \mathrm{kGy}$, respectively). After 3 weeks of feeding, hens in each group were slaughtered and flesh (breast and thigh muscles) and organs (liver, kidney, spleen and heart) were dissected out and dried, grinded and stored frozen as prepared meals $\left(M_{1}, M_{2}, M_{3}, M_{4}\right.$ and $M_{5}$, respectively) for the experimental rats. A total number of 150 adult female Westar rats, aged 90 days, weighing around $120 \pm 5 \mathrm{gm}$, were divided into five groups of 30 rats each. All groups were adapted for one week under normal ration before starting the 
experiment and then fasted for $24 \mathrm{~h}$ before feeding with the prepared meals. The first group $\left(\mathrm{G}_{1}\right)$ was fed daily on the prepared meal $\left(\mathrm{M}_{1}\right)$ of the first control group of hens (non-contaminated ration). The second group of rats $\left(\mathrm{G}_{2}\right)$ was fed on $\mathrm{M}_{2}$ meal of the second group of hens $\left(\mathrm{AFB}_{1}\right.$ contaminated ration). The third to fifth groups $\left(\mathrm{G}_{3}, \mathrm{G}_{4}\right.$ and $\left.\mathrm{G}_{5}\right)$ were fed on their analog prepared hens meals $\left(\mathrm{M}_{3}\right.$, $\mathrm{M}_{4}$ and $\left.\mathrm{M}_{5}\right)\left(\mathrm{AFB}_{1}\right.$ contaminated irradiated ration with 10,20 and $30 \mathrm{kGy}$, respectively). The feeding continued for 3 weeks (Experimental duration) and then followed by another 3 weeks period on commercial non-contaminated ration (Recovery duration) to explore the possibility of reducing and a polished the residue of $\mathrm{AFB}_{1}$ that may be present in the experimental rats. Indices as feed intake and live body weight of all rats were recorded weekly during the experimental and recovery periods. Three rats in each group were killed (slaughtered) as representative samples and their internal organs (liver, kidney, spleen and heart) were immediately dissected out and weighed. The $\mathrm{AFB}_{1}$ residues in these samples were measured by HPLC according AOAC (1995).

\section{Blood sampling and hormones assay}

Blood samples were collected weekly, 5 rats were chosen randomly in each group. Serum total protein, albumin, globulin, liver enzymes (AST, ALT \& ALP) and creatinine were measured by spectrophotometer, using available commercial kits produced by Biodiagnostic, Egypt. Blood serum samples of rats at the end of experimental and recovery duration (the $3^{\text {rd }}$ and the $6^{\text {th }}$ weeks) were tested for hormones determination. All tested hormones were performed by enzymelinked immunosorbent assay (ELISA) according to manufacturer instructions. Thyroid hormones; triiodothyronine $\left(\mathrm{T}_{3}\right)$, thyroxine $\left(\mathrm{T}_{4}\right)$ and thyroid stimulating hormone (TSH) and progesterone were determined using kits purchased from Immunospec Corporation. Testosterone and estradiol were determined using kits purchased from Diagnostic Biochem CandaInc .

\section{Statistical analysis}

Data were analyzed for all variables using the General Linear Models procedure to test the differences between means using SAS software version 9.1 (SAS Institute, 2004). Means showing significant differences were compared using Duncan's Multiple Range procedure (Duncan, 1955). All statements of statistical significance were based on probability $\mathrm{P}<0.05$.

\section{Results and Discussion}

\section{Feed intake and live body weight}

The weekly feed intake and the live body weight of rats during the experimental and recovery durations are presented in Table 1 . These indices were severely depressed in rats in $\mathrm{G}_{2}$ during the experimental duration $\left(1^{\text {st }}-3^{\text {rd }}\right.$ week) compared to those in $\mathrm{G}_{3}, \mathrm{G}_{4}$ and $\mathrm{G}_{5}$. On the other hand, these values were increased gradually by the rate of irradiation to reach the same values of the control group $\left(\mathrm{G}_{1}\right)$.

These results are in agreement with El-Shewy \& Ebrahem (2004), who reported a significant decrease in body weight and growth rate of rats dosed $\mathrm{AFB}_{1}(7.5 \mathrm{ug} / 200 \mathrm{~g}$ body weight orally) daily for 3 successive weeks $(26.84 \%)$ than the control group (53.55\%). The loss of body weight may be due to improper assimilation or metabolism of feed produced from the hepatotoxic effects of $\mathrm{AFB}_{1}$ (Ibeh \& Saxena, 1998). In addition, lower feed intake and growth rate might be due to the decreased activity of important enzymes in the carbohydrates digestion, proteins, lipids, and nucleic acids and impaired and defects in some of the nutrient. The reduction in protein synthesis was affected by AFs may due to disruption of transcription mRNA and transport amino acid, thus protein synthesis and DNA were prevented (Thaxton et al., 1974). The improvements in feed intake and body weight gain by irradiation treatments of $\mathrm{AFB}_{1}$ - contaminated diets may due to residual degradation of $\mathrm{AFB}_{1}$ levels in diets which was reflected in laying hens' tissues and organs and the degradation level increased with increasing the level of $\gamma$-radiation. In the recovery phase $\left(4^{\text {th }}, 5^{\text {th }}\right.$ and $6^{\text {th }}$ weeks), a slight improvement of these indices was recorded in $G_{2}$, with a significant increase $(P<0.05)$ in $G_{3}$, $\mathrm{G}_{4}$ and $\mathrm{G}_{5}$ (Table 1).

\section{Relative organ weights}

The relative weights of some organs including liver, kidneys, spleen and heart to live weights of rats in different groups are presented in Table 2. The relative weight of these organs in $\mathrm{G}_{2}$ were significantly increased $(\mathrm{P}<0.05)$ compared to those of the control $\left(G_{1}\right)$. The effect was directly proportion with the duration. Treatment of the laying hens' diet with $\gamma$-radiation at different doses up to $30 \mathrm{kGy}$ reduced the toxic effects of $\mathrm{AFB}_{1}$ on the relative weights of rats' organs. It is concluded that $\gamma$ - irradiation at dose level of $30 \mathrm{kGy}$ normalized the relative weight of 
organs in corresponding to the control group. Increased liver weight was probably due to the fat deposition consequently to impair fat metabolism. Liver is considered the target organ for $\mathrm{AFB}_{1}$ because it is the organ where most aflatoxins are bioactivated to the reactive 8,9 -epoxide form, which is known to bind DNA and proteins, damaging the liver structures and increasing liver weight (Pasha et al., 2007). Another explanation for increased weight of liver, fatty liver is mainly mediated by inhibiting synthesis of cholesterol and phospholipids, hence the fat transported through hepatic tissue (Manegar et al., 2010).
In addition to the irritation of gastrointestinal mucosa by aflatoxins, provoking inflammation and thickening of the wall (El-Ghany et al., 2013). Increased relative weight of kidneys was probably due to lipaemia (increased fat deposition) (Sharghi \& Manafi, 2011). Lower relative weights of the liver, spleen and kidneys in rats challenged with only AFs compared to untreated control rats could be due to necrosis and reduced density of lymphoid cells (Perozo \& Rivera, 2003). During the recovery period $\left(4^{\text {th }}-6^{\text {th }}\right.$ week $)$, the relative weight of the rat organs in $\mathrm{G}_{5}$ reached the nearest normal weights of the control group $\left(\mathrm{G}_{1}\right)$.

TABLE 1. Feed intake and live body weight of the experimental rats in different treated groups (T) and during experimental duration (D) and recovery duration (R).

\begin{tabular}{|c|c|c|c|c|}
\hline $\begin{array}{l}\text { Duration } \\
\text { (week) }\end{array}$ & & $\begin{array}{c}\text { Experimental } \\
\text { treated groups } \\
(\mathrm{T}) \\
\end{array}$ & $\begin{array}{c}\text { Feed } \\
\text { intake } \\
(\mathrm{g}) \\
\end{array}$ & $\begin{array}{l}\text { Live body } \\
\text { weight } \\
\text { (g) } \\
\end{array}$ \\
\hline \multirow{15}{*}{$\begin{array}{l}\text { Experimental } \\
\text { duration } \\
\text { (D) }\end{array}$} & \multirow{9}{*}{$1^{\text {st }}$} & $\mathrm{G}_{1}$ & $222.30^{\mathrm{a}} \pm 1.28$ & $134.00^{\mathrm{a}} \pm 1.15$ \\
\hline & & $\mathrm{G}_{2}$ & $117.63^{\mathrm{e}} \pm 3.1$ & $116.33^{\mathrm{d}} \pm 1.45$ \\
\hline & & $\mathrm{G}_{3}$ & $123.86^{\mathrm{d}} \pm 1.29$ & $121.66^{\mathrm{c}} \pm 0.88$ \\
\hline & & $\mathrm{G}_{4}$ & $143.30^{\mathrm{c}} \pm 0.75$ & $126.66^{\mathrm{b}} \pm 0.33$ \\
\hline & & $\mathrm{G}_{5}$ & $162.40^{\mathrm{b}} \pm 0.43$ & $131.66^{\mathrm{a}} \pm 0.88$ \\
\hline & & $\mathrm{G}_{1}$ & $246.00^{\mathrm{a}} \pm 0.96$ & $144.33^{\mathrm{a}} \pm 1.76$ \\
\hline & & $\mathrm{G}_{2}$ & $117.70^{\mathrm{d}} \pm 1.48$ & $114.66^{\mathrm{d}} \pm 0.81$ \\
\hline & & $\mathrm{G}_{3}$ & $117.40^{\mathrm{d}} \pm 2.75$ & $118.66^{\mathrm{c}} \pm 0.85$ \\
\hline & & $\mathrm{G}_{4}^{3}$ & $129.03^{\mathrm{c}} \pm 0.33$ & $121.33^{\mathrm{c}} \pm 0.66$ \\
\hline & \multirow{6}{*}{$3^{\text {rd }}$} & $\mathrm{G}_{5}^{4}$ & $135.53^{\mathrm{b}} \pm 0.57$ & $127.66^{b} \pm 0.66$ \\
\hline & & $\mathrm{G}_{1}$ & $263.56^{\mathrm{a}} \pm 1.18$ & $151.00^{\mathrm{a}} \pm 0.57$ \\
\hline & & $\mathrm{G}_{2}$ & $118.40^{\mathrm{d}} \pm 0.64$ & $114.00^{\mathrm{d}} \pm 1.00$ \\
\hline & & $\mathrm{G}_{3}^{2}$ & $119.26^{\mathrm{d}} \pm 1.26$ & $118.33^{\mathrm{c}} \pm 0.33$ \\
\hline & & $\mathrm{G}_{4}$ & $224.80^{c} \pm 2.59$ & $120.66^{b} \pm 0.33$ \\
\hline & & $\mathrm{G}_{5}$ & $230.93^{b} \pm 0.27$ & $122.33^{\mathrm{b}} \pm 0.88$ \\
\hline \multirow{14}{*}{$\begin{array}{l}\text { Recovery } \\
\text { duration } \\
(\mathrm{R})\end{array}$} & \multirow{4}{*}{$4^{\text {th }}$} & \multirow{14}{*}{$\begin{array}{l}\text { Commercial non- } \\
\text { contaminated diet with } \\
\mathrm{AFB}_{1}\end{array}$} & $\begin{array}{l}274.43^{\mathrm{a}} \pm 0.91 \\
123.60^{\mathrm{e}} \pm 2.23\end{array}$ & $\begin{array}{l}159.33^{\mathrm{a}} \pm 0.88 \\
119.00^{\mathrm{e}} \pm 0.57\end{array}$ \\
\hline & & & $133.20^{\mathrm{d}} \pm 2.85$ & $125.33^{\mathrm{d}} \pm 0.88$ \\
\hline & & & $144.90^{\mathrm{c}} \pm 1.85$ & $129.33^{\mathrm{c}} \pm 0.66$ \\
\hline & & & $156.36^{\mathrm{b}} \pm 2.60$ & $134.00^{\mathrm{b}} \pm 1.52$ \\
\hline & \multirow{6}{*}{$5^{\text {th }}$} & & $291.33^{\mathrm{a}} \pm 1.99$ & $165.66^{\mathrm{a}} \pm 1.20$ \\
\hline & & & $139.43^{\mathrm{e}} \pm 1.75$ & $126.33^{\mathrm{e}} \pm 1.20$ \\
\hline & & & $159.03^{\mathrm{d}} \pm 4.27$ & $132.33^{\mathrm{d}} \pm 1.45$ \\
\hline & & & $173.03^{\mathrm{c}} \pm 1.03$ & $137.66^{\mathrm{c}} \pm 1.33$ \\
\hline & & & $187.36^{\mathrm{b}} \pm 0.95$ & $148.33^{b} \pm 0.66$ \\
\hline & & & $289.43^{\mathrm{a}} \pm 4.67$ & $176.00^{\mathrm{a}} \pm 1.15$ \\
\hline & \multirow{4}{*}{$6^{\text {th }}$} & & $157.63^{\mathrm{e}} \pm 1.54$ & $137.00^{\mathrm{e}} \pm 0.57$ \\
\hline & & & $177.20^{\mathrm{d}} \pm 1.27$ & $145.33^{\mathrm{d}} \pm 2.33$ \\
\hline & & & $189.73^{\mathrm{c}} \pm 1.56$ & $157.00^{\mathrm{c}} \pm 1.52$ \\
\hline & & & $227.13^{b} \pm 0.23$ & $162.00^{b} \pm 1.52$ \\
\hline
\end{tabular}

Means of each value in the same column within each duration with the same letter are not significantly different $(\mathrm{P}<0.05)$. Each value represents mean \pm standard error. The P value of Duration (D), Treatment (T), D*T, Recovery (R), Treatment (T), R*T was 0.0001 . 
TABLE 2. Relative organ weights of the experimental rats in different treated groups $(T)$ and during experimental duration (D) and recovery duration (R).

\begin{tabular}{|c|c|c|c|c|c|c|c|}
\hline $\begin{array}{l}\text { Duration } \\
\text { (week) }\end{array}$ & & $\begin{array}{c}\text { Experimental } \\
\text { treated groups } \\
\text { (T) }\end{array}$ & $\begin{array}{l}\text { Live body } \\
\text { weight } \\
\text { (g) }\end{array}$ & $\begin{array}{c}\text { Liver } \\
\text { (g/100g live } \\
\text { body weight) }\end{array}$ & $\begin{array}{c}\text { Kidney } \\
\text { (g/100g live } \\
\text { body weight) }\end{array}$ & $\begin{array}{c}\text { Spleen } \\
\text { (g/100g live } \\
\text { body weight) }\end{array}$ & $\begin{array}{c}\text { Heart } \\
\text { (g/100g of } \\
\text { live body } \\
\text { weight) } \\
\end{array}$ \\
\hline \multirow{15}{*}{$\begin{array}{l}\text { Experimental } \\
\text { duration } \\
\text { (D) }\end{array}$} & \multirow{5}{*}{$1^{\mathrm{st}}$} & $\mathrm{G}_{1}$ & $146.33^{\mathrm{a}} \pm 2.18$ & $3.26^{\mathrm{e}} \pm 0.02$ & $1.49^{\mathrm{d}} \pm 0.04$ & $0.68^{\mathrm{d}} \pm 0.01$ & $0.75^{\mathrm{d}} \pm 0.02$ \\
\hline & & $\mathrm{G}_{2}$ & $122.33^{\mathrm{d}} \pm 0.88$ & $4.27^{\mathrm{a}} \pm 0.01$ & $1.90^{\mathrm{a}} \pm 0.01$ & $1.19^{\mathrm{a}} \pm 0.01$ & $1.19^{\mathrm{a}} \pm 0.02$ \\
\hline & & $\mathrm{G}_{3}$ & $127.00^{\mathrm{cd}} \pm 1.52$ & $4.06^{\mathrm{b}} \pm 0.02$ & $1.80^{\mathrm{b}} \pm 0.04$ & $1.12^{\mathrm{b}} \pm 0.01$ & $1.10^{\mathrm{b}} \pm 0.01$ \\
\hline & & $\mathrm{G}_{4}$ & $131.33^{\mathrm{c}} \pm 2.60$ & $3.82^{\mathrm{c}} \pm 0.03$ & $1.76^{\mathrm{b}} \pm 0.01$ & $1.0^{\mathrm{b}} \pm 0.02$ & $1.05^{\mathrm{b}} \pm 0.01$ \\
\hline & & $\mathrm{G}_{5}$ & $137.00^{\mathrm{b}} \pm 1.15$ & $3.54^{\mathrm{d}} \pm 0.02$ & $1.62^{\mathrm{c}} \pm 0.01$ & $0.98^{\mathrm{c}} \pm 0.03$ & $0.86^{\mathrm{c}} \pm 0.02$ \\
\hline & \multirow{5}{*}{$2^{\text {nd }}$} & $\mathrm{G}_{1}$ & $149.66^{\mathrm{a}} \pm 2.40$ & $3.30^{\mathrm{e}} \pm 0.02$ & $1.47^{\mathrm{e}} \pm 0.02$ & $0.66^{\mathrm{c}} \pm 0.02$ & $0.68^{\mathrm{e}} \pm 0.01$ \\
\hline & & $\mathrm{G}_{2}$ & $114.66^{\mathrm{d}} \pm 0.88$ & $4.32^{\mathrm{a}} \pm 0.02$ & $1.95^{\mathrm{a}} \pm 0.02$ & $1.28^{\mathrm{a}} \pm 0.01$ & $1.27^{\mathrm{a}} \pm 0.01$ \\
\hline & & $\mathrm{G}_{3}$ & $117.66^{\mathrm{cd}} \pm 0.66$ & $4.16^{\mathrm{b}} \pm 0.01$ & $1.82^{\mathrm{b}} \pm 0.01$ & $1.21^{\mathrm{ab}} \pm 0.06$ & $1.19^{\mathrm{b}} \pm 0.01$ \\
\hline & & $\mathrm{G}_{4}$ & $121.33^{\mathrm{c}} \pm 0.66$ & $3.86^{\mathrm{c}} \pm 0.06$ & $1.74^{\mathrm{c}} \pm 0.02$ & $1.17^{\mathrm{ab}} \pm 0.01$ & $1.11^{\mathrm{c}} \pm 0.01$ \\
\hline & & $\mathrm{G}_{5}$ & $127.00^{\mathrm{b}} \pm 1.15$ & $3.67^{\mathrm{d}} \pm 0.02$ & $1.66^{\mathrm{d}} \pm 0.02$ & $1.11^{\mathrm{b}} \pm 0.01$ & $0.88^{\mathrm{d}} \pm 0.02$ \\
\hline & \multirow{5}{*}{$3^{\text {rd }}$} & $\mathrm{G}_{1}$ & $153.66^{\mathrm{a}} \pm 2.84$ & $3.31^{\mathrm{e}} \pm 0.02$ & $1.40^{\mathrm{d}} \pm 0.05$ & $0.67^{\mathrm{c}} \pm 0.04$ & $0.69^{\mathrm{c}} \pm 0.04$ \\
\hline & & $\mathrm{G}_{2}$ & $114.00^{\mathrm{c}} \pm 1.00$ & $4.40^{\mathrm{a}} \pm 0.02$ & $1.93^{\mathrm{a}} \pm 0.03$ & $1.33^{\mathrm{a}} \pm 0.03$ & $1.31^{\mathrm{a}} \pm 0.01$ \\
\hline & & $\mathrm{G}_{3}$ & $118.33^{\mathrm{bc}} \pm 0.33$ & $4.24^{b} \pm 0.01$ & $1.84^{\mathrm{ab} \pm 0.01}$ & $1.28^{\mathrm{ab} \pm 0.04}$ & $1.24^{\mathrm{a}} \pm 0.01$ \\
\hline & & $\mathrm{G}_{4}$ & $120.33^{b} \pm 0.33$ & $3.88^{c} \pm 0.04$ & $1.78^{\mathrm{b}} \pm 0.01$ & $1.21^{\mathrm{b}} \pm 0.01$ & $1.16^{\mathrm{b}} \pm 0.01$ \\
\hline & & $\mathrm{G}_{5}$ & $122.33^{b} \pm 0.88$ & $3.64 \mathrm{~d} \pm 0.02$ & $1.69^{\mathrm{c}} \pm 0.01$ & $0.67^{c} \pm 0.01$ & $0.93^{\mathrm{c}} \pm 0.02$ \\
\hline \multirow{15}{*}{$\begin{array}{l}\text { Recovery } \\
\text { duration } \\
\text { (R) }\end{array}$} & \multirow{5}{*}{$4^{\text {th }}$} & \multirow{15}{*}{$\begin{array}{l}\text { Commercial } \\
\text { non- } \\
\text { contaminated } \\
\text { diet with AFB1 }\end{array}$} & $157.00^{\mathrm{a}} \pm 3.05$ & $3.35^{\mathrm{e}} \pm 0.02$ & $1.43^{\mathrm{e}} \pm 0.03$ & $0.69^{c} \pm 0.01$ & $0.71^{\mathrm{e}} \pm 0.01$ \\
\hline & & & $119.00^{\mathrm{d}} \pm 0.57$ & $4.30^{\mathrm{a}} \pm 0.01$ & $1.85^{\mathrm{a}} \pm 0.01$ & $1.23^{\mathrm{a}} \pm 0.04$ & $1.21^{\mathrm{a}} \pm 0.01$ \\
\hline & & & $125.33^{\mathrm{c}} \pm 0.88$ & $4.13^{b} \pm 0.01$ & $1.74^{\mathrm{b}} \pm 0.01$ & $1.22^{\mathrm{a}} \pm 0.03$ & $1.15^{b} \pm 0.01$ \\
\hline & & & $129.33^{b c} \pm 0.66$ & $3.67^{\mathrm{c}} \pm 0.02$ & $1.67^{\mathrm{c}} \pm 0.02$ & $1.13^{\mathrm{a}} \pm 0.02$ & $1.02^{\mathrm{c}} \pm 0.01$ \\
\hline & & & $134.00^{\mathrm{b}} \pm 1.52$ & $3.55^{\mathrm{d}} \pm 0.01$ & $1.59^{\mathrm{d}} \pm 0.01$ & $0.97^{b} \pm 0.03$ & $0.80^{\mathrm{d}} \pm 0.01$ \\
\hline & \multirow{5}{*}{$5^{\text {th }}$} & & $160.33^{\mathrm{a}} \pm 1.76$ & $3.33^{\mathrm{e}} \pm 0.03$ & $1.39^{\mathrm{e}} \pm 0.01$ & $0.65^{\mathrm{e}} \pm 0.02$ & $0.68^{\mathrm{d}} \pm 0.01$ \\
\hline & & & $126.33^{\mathrm{e}} \pm 1.20$ & $4.20^{\mathrm{a}} \pm 0.01$ & $1.76^{\mathrm{a}} \pm 0.01$ & $1.09^{\mathrm{a}} \pm 0.02$ & $1.12^{\mathrm{a}} \pm 0.03$ \\
\hline & & & $132.33^{\mathrm{d}} \pm 1.45$ & $3.93^{b} \pm 0.02$ & $1.66^{\mathrm{b}} \pm 0.01$ & $0.97^{b} \pm 0.02$ & $1.03^{\mathrm{b}} \pm 0.01$ \\
\hline & & & $137.66^{\mathrm{c}} \pm 1.33$ & $3.61^{\mathrm{c}} \pm 0.02$ & $1.56^{\mathrm{c}} \pm 0.01$ & $0.83^{\mathrm{c}} \pm 0.01$ & $0.93^{\mathrm{c}} \pm 0.01$ \\
\hline & & & $148.33^{b} \pm 0.66$ & $3.44^{\mathrm{d}} \pm 0.01$ & $1.48^{\mathrm{d}} \pm 0.02$ & $0.73^{\mathrm{d}} \pm 0.01$ & $0.73^{\mathrm{d}} \pm 0.01$ \\
\hline & \multirow{5}{*}{$6^{\text {th }}$} & & $173.00^{\mathrm{a}} \pm 2.08$ & $3.31^{\mathrm{d}} \pm 0.02$ & $1.39^{\mathrm{d}} \pm 0.01$ & $0.65^{\mathrm{d}} \pm 0.01$ & $0.65^{\mathrm{d}} \pm 0.01$ \\
\hline & & & $133.33^{\mathrm{d}} \pm 0.88$ & $3.89^{\mathrm{a}} \pm 0.03$ & $1.66^{\mathrm{a}} \pm 0.01$ & $0.91^{\mathrm{a}} \pm 0.01$ & $0.95^{\mathrm{a}} \pm 0.01$ \\
\hline & & & $148.00^{\mathrm{c}} \pm 2.30$ & $3.77^{b} \pm 0.02$ & $1.57^{\mathrm{b}} \pm 0.01$ & $0.82^{\mathrm{b}} \pm 0.01$ & $0.88^{\mathrm{b}} \pm 0.01$ \\
\hline & & & $157.00^{\mathrm{b}} \pm 1.52$ & $3.54^{\mathrm{c}} \pm 0.01$ & $1.48^{\mathrm{c}} \pm 0.03$ & $0.74^{c} \pm 0.02$ & $0.76^{\mathrm{c}} \pm 0.01$ \\
\hline & & & $168.66^{\mathrm{a}} \pm 1.85$ & $3.34^{\mathrm{d}} \pm 0.01$ & $1.39^{\mathrm{d}} \pm 0.01$ & $0.67^{\mathrm{d}} \pm 0.02$ & $0.67^{\mathrm{d}} \pm 0.01$ \\
\hline
\end{tabular}

Means of value sample in the same column within each duration with the same letter are not significantly different $(\mathrm{P}<0.05)$. Each value represents mean \pm standard error. The P value of Duration (D), Treatment (T), D*T, Recovery (R), Treatment (T), R*T was 0.0001 . 
$A F B_{1}$ residues in organs

The detectable amount of $\mathrm{AFB}_{1}$ residues in the rats breast tissues and organs of $G_{2}$ were significantly higher $(P<0.05)$ than those in $G_{1}$, $\mathrm{G}_{3}, \mathrm{G}_{4}$ and $\mathrm{G}_{5}$ (Table 3). In general, $\mathrm{AFB}_{1}$ residues were decreased dramatically in these organs in parallel with elevation of the radiation dose up to $30 \mathrm{kGy}$. It is concluded that the residual levels of $\mathrm{AFB}_{1}$ were diminished by $\gamma$-radiation processing of contaminated diets, as a physical detoxifying agent. These results are agreed with Zaghini et al. (2005) who reported that $\mathrm{AFB}_{1}$ residue was found in the livers of laying hens fed $2.5 \mathrm{mg} / \mathrm{kg} \mathrm{AFB}_{1}$ diet for four weeks. Residue levels may be different owing to the type of animal and diet, however, the concentrations of $\mathrm{AFB}_{1}$ and the length of exposure, and enhanced tolerance to AFs (Bennett $\&$ Klich, 2003). During the recovery phase, $\mathrm{AFB}_{1}$ residues in different organs were decreased with increasing the duration especially for those rats in groups $\left(\mathrm{G}_{3}, \mathrm{G}_{4}\right.$ and $\left.\mathrm{G}_{5}\right)$ AFB 1 residual in breast tissues and organs until there were no detectable residues could be seen.

TABLE 3. AFB 1 residues content in breast tissues and organs of the experimental rats in different treated groups $(T)$ and during experimental duration (D) and recovery duration (R).

\begin{tabular}{|c|c|c|c|c|c|c|c|}
\hline $\begin{array}{l}\text { Duration } \\
\text { (week) }\end{array}$ & & $\begin{array}{c}\text { Experimental } \\
\text { treated } \\
\text { groups } \\
(\mathrm{T})\end{array}$ & $\begin{array}{l}\text { Breast tissues } \\
\quad\left(n g . g^{-1}\right)\end{array}$ & $\begin{array}{l}\text { Liver } \\
\left(\text { ng.g }^{-1}\right)\end{array}$ & $\begin{array}{l}\text { Kidney } \\
\left(\text { ng.g }^{-1}\right)\end{array}$ & $\begin{array}{l}\text { Spleen } \\
(\text { ng.g } \\
\end{array}$ & $\begin{array}{l}\text { Heart } \\
(\text { ng.g } \\
\text { (n) }\end{array}$ \\
\hline \multirow{15}{*}{$\begin{array}{l}\text { Experimental } \\
\text { duration } \\
\text { (D) }\end{array}$} & \multirow{5}{*}{$1^{\text {st }}$} & G1 & ND & ND & ND & ND & ND \\
\hline & & $\mathrm{G}_{2}$ & $0.80^{\mathrm{a}} \pm 0.04$ & $0.91^{\mathrm{a}} \pm 0.01$ & $0.76^{\mathrm{a}} \pm 0.02$ & $0.75^{\mathrm{a}} \pm 0.01$ & $0.85^{\mathrm{a}} \pm 0.01$ \\
\hline & & $\mathrm{G}_{3}^{2}$ & $0.66^{b} \pm 0.02$ & $0.80^{\mathrm{b}} \pm 0.03$ & $0.65^{b} \pm 0.02$ & $0.68^{\mathrm{a}} \pm 0.03$ & $0.73^{b} \pm 0.01$ \\
\hline & & $\mathrm{G}_{4}$ & $0.55^{\mathrm{c}} \pm 0.02$ & $0.72^{\mathrm{c}} \pm 0.01$ & $0.45^{\mathrm{c}} \pm 0.02$ & $0.52^{b} \pm 0.03$ & $0.60^{c} \pm 0.01$ \\
\hline & & $\mathrm{G}_{5}$ & $0.48^{c} \pm 0.02$ & $0.65^{\mathrm{c}} \pm 0.02$ & $0.32^{\mathrm{d}} \pm 0.02$ & $0.31^{\mathrm{c}} \pm 0.02$ & $0.31^{\mathrm{d}} \pm 0.01$ \\
\hline & \multirow{5}{*}{$2^{\text {nd }}$} & $\mathrm{G}_{1}$ & ND & ND & ND & ND & ND \\
\hline & & $\mathrm{G}_{2}$ & $1.04^{\mathrm{a}} \pm 0.07$ & $1.26^{\mathrm{a}} \pm 0.01$ & $0.96^{\mathrm{a}} \pm 0.01$ & $0.86^{\mathrm{a}} \pm 0.03$ & $1.15^{\mathrm{a}} \pm 0.01$ \\
\hline & & $\mathrm{G}_{3}$ & $0.88^{\mathrm{b}} \pm 0.02$ & $1.11^{\mathrm{b}} \pm 0.01$ & $0.84^{\mathrm{b}} \pm 0.03$ & $0.75^{b} \pm 0.02$ & $0.89^{b} \pm 0.02$ \\
\hline & & $\mathrm{G}_{4}$ & $0.75^{\mathrm{bc}} \pm 0.02$ & $0.87^{\mathfrak{c}} \pm 0.01$ & $0.68^{\mathrm{c}} \pm 0.02$ & $0.65^{\mathrm{c}} \pm 0.02$ & $0.69^{c} \pm 0.03$ \\
\hline & & $\mathrm{G}_{5}^{4}$ & $0.66^{\mathrm{c}} \pm 0.01$ & $0.75^{\mathrm{d}} \pm 0.02$ & $0.44^{\mathrm{d}} \pm 0.03$ & $0.46^{\mathrm{d}} \pm 0.02$ & $0.45^{\mathrm{d}} \pm 0.03$ \\
\hline & \multirow{5}{*}{$3^{\text {rd }}$} & $\mathrm{G}_{1}$ & ND & ND & ND & ND & ND \\
\hline & & $\mathrm{G}_{2}$ & $1.47^{\mathrm{a}} \pm 0.03$ & $4.40^{\mathrm{a}} \pm 0.72$ & $1.28^{\mathrm{a}} \pm 0.05$ & $1.05^{\mathrm{a}} \pm 0.01$ & $1.27^{\mathrm{a}} \pm 0.04$ \\
\hline & & $\mathrm{G}_{3}$ & $1.28^{\mathrm{b}} \pm 0.03$ & $2.61^{b} \pm 0.23$ & $1.05^{\mathrm{b}} \pm 0.02$ & $0.89^{b} \pm 0.01$ & $1.10^{\mathrm{b}} \pm 0.03$ \\
\hline & & $\mathrm{G}_{4}$ & $1.13^{\mathrm{c}} \pm 0.03$ & $1.35^{\mathrm{c}} \pm 0.06$ & $0.80^{\mathrm{c}} \pm 0.01$ & $0.76^{\mathrm{c}} \pm 0.02$ & $0.83^{\mathrm{c}} \pm 0.03$ \\
\hline & & $\mathrm{G}_{5}$ & $0.89^{\mathrm{d}} \pm 0.04$ & $1.21^{\mathrm{c}} \pm 0.02$ & $0.57^{\mathrm{d}} \pm 0.02$ & $0.65^{\mathrm{d}} \pm 0.02$ & $0.62^{\mathrm{d}} \pm 0.02$ \\
\hline \multirow{15}{*}{$\begin{array}{l}\text { Recovery } \\
\text { duration } \\
\text { (R) }\end{array}$} & \multirow{5}{*}{$4^{\text {th }}$} & \multirow{15}{*}{$\begin{array}{l}\text { Commercial } \\
\text { non- } \\
\text { contaminated } \\
\text { diet with } \\
\text { AFB1 }\end{array}$} & ND & ND & ND & ND & ND \\
\hline & & & $1.26^{\mathrm{a}} \pm 0.02$ & $2.52^{\mathrm{a}} \pm 0.05$ & $0.61^{\mathrm{a}} \pm 0.03$ & $0.88^{\mathrm{a}} \pm 0.01$ & $0.98 \mathrm{a} \pm 0.07$ \\
\hline & & & $1.15^{\mathrm{b}} \pm 0.03$ & $2.23^{\mathrm{b}} \pm 0.01$ & $0.38^{\mathrm{b}} \pm 0.04$ & $0.79^{b} \pm 0.01$ & $0.80^{\mathrm{b}} \pm 0.03$ \\
\hline & & & $0.92^{\mathrm{c}} \pm 0.02$ & $1.20^{\mathrm{c}} \pm 0.01$ & $0.24^{\mathrm{c}} \pm 0.02$ & $0.57^{\mathrm{c}} \pm 0.02$ & $0.61^{\mathrm{c}} \pm 0.03$ \\
\hline & & & $0.70^{\mathrm{d}} \pm 0.02$ & $0.94^{\mathrm{d}} \pm 0.03$ & $0.15^{\mathrm{d}} \pm 0.02$ & $0.38^{\mathrm{d}} \pm 0.02$ & $0.36^{\mathrm{d}} \pm 0.02$ \\
\hline & \multirow{5}{*}{$5^{\text {th }}$} & & ND & ND & ND & ND & ND \\
\hline & & & $1.07^{\mathrm{a}} \pm 0.02$ & $1.64^{\mathrm{a}} \pm 0.05$ & $0.44^{\mathrm{a}} \pm 0.01$ & $0.74^{\mathrm{a}} \pm 0.02$ & $1.10^{\mathrm{a}} \pm 0.05$ \\
\hline & & & $0.78^{\mathrm{b}} \pm 0.05$ & $1.37^{\mathrm{b}} \pm 0.02$ & $0.33^{b} \pm 0.03$ & $0.63^{b} \pm 0.02$ & $0.90^{b} \pm 0.02$ \\
\hline & & & $0.58^{\mathrm{c}} \pm 0.02$ & $0.89^{\mathrm{c}} \pm 0.01$ & $0.16^{\mathrm{c}} \pm 0.01$ & $0.43^{\mathrm{c}} \pm 0.01$ & $0.72^{\mathrm{c}} \pm 0.02$ \\
\hline & & & ND & $0.27^{\mathrm{d}} \pm 0.02$ & ND & ND & $0.17^{\mathrm{d}} \pm 0.03$ \\
\hline & \multirow{5}{*}{$6^{\text {th }}$} & & ND & ND & ND & ND & ND \\
\hline & & & $0.50^{\mathrm{a}} \pm 0.01$ & $0.72^{\mathrm{a}} \pm 0.04$ & $0.32^{\mathrm{a}} \pm 0.01$ & $0.46^{\mathrm{a}} \pm 0.02$ & $0.68^{a} \pm 0.05$ \\
\hline & & & $0.42^{b} \pm 0.03$ & $0.63^{b} \pm 0.03$ & $0.20^{\mathrm{b}} \pm 0.01$ & $0.31^{\mathrm{b}} \pm 0.05$ & $0.51^{\mathrm{b}} \pm 0.04$ \\
\hline & & & $0.20^{c} \pm 0.02$ & $0.46^{\mathrm{c}} \pm 0.02$ & ND & $0.16^{\mathrm{c}} \pm 0.01$ & $0.20^{c} \pm 0.04$ \\
\hline & & & ND & ND & ND & ND & ND \\
\hline
\end{tabular}

-Means of value sample in the same column within each duration with the same letter are not significantly different $(\mathrm{P}<0.05)$. Each value represents mean \pm standard error. The P value of Duration (D), Treatment $(T), D^{*} T$, Recovery(R), Treatment (T), R*T was 0.0001 . -ND: no detectable. 
Serum biochemical attributes

It is well known that, AFB residue in hen's meals has a harmful and stressful effect on liver tissue. AST, ALT and ALP enzymes are famous biomarkers of liver damage. The results of the current study revealed that exposure to $\mathrm{AFB}_{1}$ residues resulted in a significant increase in AST, ALT and ALP (Table 4).

TABLE 4. Liver enzymes of the experimental rats in different treated groups $(T)$ and during experimental duration (D) and recovery duration $(\mathrm{R})$.

\begin{tabular}{|c|c|c|c|c|c|}
\hline $\begin{array}{l}\text { Duration } \\
\text { (week) }\end{array}$ & & $\begin{array}{l}\text { Experimental } \\
\text { treated groups } \\
\text { (T) }\end{array}$ & $\begin{array}{l}\text { AST } \\
\text { (UL) }\end{array}$ & $\begin{array}{l}\text { ALT } \\
\text { (UL) }\end{array}$ & $\begin{array}{l}\text { ALP } \\
\text { (UL) }\end{array}$ \\
\hline \multirow{15}{*}{$\begin{array}{l}\text { Experimental } \\
\text { duration } \\
\text { (D) }\end{array}$} & \multirow{5}{*}{$1^{\text {st }}$} & $\mathrm{G}_{1}$ & $37.73^{\mathrm{e}} \pm 1.26$ & $56.48^{\mathrm{e}} \pm 1.96$ & $27.86^{\mathrm{e}} \pm 1.28$ \\
\hline & & $\mathrm{G}_{2}$ & $81.34^{\mathrm{a}} \pm 0.68$ & $93.62^{\mathrm{a}} \pm 2.52$ & $56.95^{\mathrm{a}} \pm 1.17$ \\
\hline & & $\mathrm{G}_{3}$ & $73.34^{\mathrm{b}} \pm 2.52$ & $86.12^{b} \pm 1.13$ & $49.64^{b} \pm 0.60$ \\
\hline & & $\mathrm{G}_{4}$ & $60.38^{\mathrm{c}} \pm 1.65$ & $80.23^{\mathrm{c}} \pm 0.88$ & $42.79^{c} \pm 0.96$ \\
\hline & & $\mathrm{G}_{5}$ & $45.38^{\mathrm{d}} \pm 2.24$ & $64.45 \mathrm{~d} \pm 2.20$ & $38.88^{\mathrm{d}} \pm 0.50$ \\
\hline & \multirow{5}{*}{$2^{\text {nd }}$} & $\mathrm{G}_{1}$ & $38.37^{\mathrm{d}} \pm 0.60$ & $53.98^{\mathrm{d}} \pm 0.33$ & $26.97^{\mathrm{e}} \pm 1.19$ \\
\hline & & $\mathrm{G}_{2}$ & $84.83^{\mathrm{a}} \pm 1.21$ & $98.43^{\mathrm{a}} \pm 1.02$ & $61.75^{\mathrm{a}} \pm 0.67$ \\
\hline & & $\mathrm{G}_{3}$ & $68.88^{\mathrm{b}} \pm 1.56$ & $79.57^{\mathrm{b}} \pm 2.28$ & $44.40^{b} \pm 1.19$ \\
\hline & & $\mathrm{G}_{4}$ & $53.45^{\mathrm{c}} \pm 2.48$ & $71.43^{\mathrm{c}} \pm 0.68$ & $39.75^{c} \pm 0.52$ \\
\hline & & $\mathrm{G}_{5}$ & $41.75^{\mathrm{d}} \pm 0.89$ & $57.21^{\mathrm{d}_{ \pm}} \pm 3.18$ & $31.62^{\mathrm{d}} \pm 0.85$ \\
\hline & \multirow{5}{*}{$3^{\text {rd }}$} & $\mathrm{G}_{1}$ & $36.50^{\mathrm{d}} \pm 0.51$ & $50.37^{\mathrm{d}} \pm 1.98$ & $25.49^{\mathrm{d}} \pm 1.14$ \\
\hline & & $\mathrm{G}_{2}$ & $77.18^{\mathrm{a}} \pm 1.11$ & $90.68^{\mathrm{a}} \pm 2.37$ & $73.60^{a} \pm 3.52$ \\
\hline & & $\mathrm{G}_{3}$ & $62.61^{\mathrm{b}} \pm 1.60$ & $75.00^{\mathrm{b}} \pm 2.35$ & $43.35^{b} \pm 1.21$ \\
\hline & & G4 & $47.15^{\mathrm{c}} \pm 0.68$ & $65.71^{\mathrm{c}} \pm 2.12$ & $34.59^{\mathrm{c}} \pm 1.65$ \\
\hline & & $\mathrm{G}_{5}$ & $38.47^{\mathrm{d}} \pm 0.68$ & $50.14^{\mathrm{d}} \pm 2.12$ & $26.80^{\mathrm{d}} \pm 1.65$ \\
\hline \multirow{15}{*}{$\begin{array}{l}\text { Recovery duration } \\
\text { (R) }\end{array}$} & \multirow{5}{*}{$1^{\text {st }}$} & \multirow{15}{*}{$\begin{array}{l}\text { Commercial non- } \\
\text { contaminated diet with } \\
\text { AFB1 }\end{array}$} & $35.11^{\mathrm{d}} \pm 1.17$ & $45.42^{\mathrm{d}} \pm 0.60$ & $25.24^{\mathrm{d}} \pm 0.34$ \\
\hline & & & $67.85^{\mathrm{a}} \pm 1.22$ & $85.78^{\mathrm{a}} \pm 2.07$ & $65.91^{\mathrm{a}} \pm 0.88$ \\
\hline & & & $56.64^{\mathrm{b}} \pm 0.87$ & $69.48^{\mathrm{b}} \pm 0.89$ & $41.88^{b} \pm 0.90$ \\
\hline & & & $47.90^{c} \pm 0.40$ & $59.49^{c} \pm 1.85$ & $33.34^{\mathrm{c}} \pm 0.47$ \\
\hline & & & $36.73^{d} \pm 0.32$ & $47.35^{\mathrm{d}} \pm 1.31$ & $25.34^{\mathrm{d}} \pm 0.49$ \\
\hline & \multirow{5}{*}{$2^{\text {nd }}$} & & $35.15^{\mathrm{d}} \pm 0.69$ & $44.16^{\mathrm{d}} \pm 0.84$ & $22.75^{\mathrm{d}} \pm 0.38$ \\
\hline & & & $62.84^{\mathrm{a}} \pm 0.79$ & $76.53^{\mathrm{a}} \pm 1.18$ & $58.52^{\mathrm{a}} \pm 1.22$ \\
\hline & & & $53.07^{b} \pm 1.15$ & $64.71^{\mathrm{b}} \pm 0.54$ & $38.59^{\mathrm{b}} \pm 1.57$ \\
\hline & & & $41.49^{\mathrm{c}} \pm 0.71$ & $54.98^{c} \pm 1.45$ & $30.76^{\mathrm{c}} \pm 0.81$ \\
\hline & & & $35.70^{\mathrm{d}} \pm 0.26$ & $44.08^{\mathrm{d}} \pm 1.36$ & $23.77^{\mathrm{d}} \pm 1.05$ \\
\hline & \multirow{5}{*}{$3^{\text {rd }}$} & & $34.28^{\mathrm{d}} \pm 0.70$ & $42.42^{\mathrm{d}} \pm 0.69$ & $23.21^{\mathrm{d}} \pm 0.30$ \\
\hline & & & $58.44^{\mathrm{a}} \pm 1.16$ & $70.93^{a} \pm 0.71$ & $53.01^{\mathrm{a}} \pm 1.39$ \\
\hline & & & $48.47^{\mathrm{b}} \pm 0.86$ & $59.32^{b} \pm 0.75$ & $36.64^{b} \pm 1.54$ \\
\hline & & & $40.31^{\mathrm{c}} \pm 0.58$ & $51.67^{c} \pm 0.58$ & $29.89^{c} \pm 0.63$ \\
\hline & & & $34.64^{\mathrm{d}} \pm 0.33$ & $42.56^{\mathrm{d}} \pm 1.06$ & $23.18^{\mathrm{d}} \pm 1.23$ \\
\hline
\end{tabular}

Means of each value in the same column within each duration with the same letter are not significantly different $(\mathrm{P}<0.05)$. Each value represents mean \pm standard error. The P value of Duration (D), Treatment (T), D*T, Recovery(R), Treatment (T), R*T was 0.0001 . 
The highest level of liver enzymes observed in $\mathrm{G}_{2}$ which were reduced with increasing levels of $\gamma$-irradiation for those rats of $\mathrm{G}_{3}, \mathrm{G}_{4}$ and $\mathrm{G}_{5}$ during the experimental duration, especially at $30 \mathrm{kGy}$ $\left(\mathrm{G}_{5}\right)$. AST and ALT are cytoplasmic in location and the increased serum levels may be due to leakage of these enzymes into blood stream as a result of autolytic breakdown or cellular damage (Gaskill et al., 2005). Elevated levels of ALT and ALP are suggestive of liver or bile duct disease, while ALT is a prime marker of bile duct epithelial proliferation that is typical of aflatoxicosis (Kramer, 1989). During the recovery duration, an improvement in liver enzymes has been observed in $\mathrm{G}_{3}, \mathrm{G}_{4}$ and $\mathrm{G}_{5}$, especially of those in group 5 . Blood serum creatinine, protein, albumin and globulin levels in different groups and during the experimental and recovery duration were recorded in Table 5. Serum creatinine value was significantly increased $(\mathrm{P}<0.05)$, while serum protein, albumin and globulin levels were significantly decreased ( $\mathrm{P}$ $<0.05)$ in rats of $\mathrm{G}_{2}$ fed on hen aflatoxicated meat.

The adverse effects were ameliorated by feeding the rats $\gamma$-irradiated hens' meal, particularly in group 5. The increase in serum creatinine level to nephrotoxic action causes renal impairment by destruction of epithelial cells of proximal and distal convoluted tubules and alteration in tubular function (Abu-Aita et al., 2008). While, the decrease in albumin and globulin values, which are commonly in association with the chronic disease (Coles, 1986) may be due to the effect of AFs on protein synthesis and RNA production (Agag, 2004). It was noticed that at the end of the recovery duration all blood parameters especially for those in $\mathrm{G}_{5}$ were improved.

\section{Blood Serum Hormone Levels}

Serum thyroid and sexual hormone values in different groups of rats at the end of the experimental and recovery duration were presented in Table 6 . Rats in $\mathrm{G}_{2}$ which consumed prepared hen's meal of $\mathrm{AFB}_{1}$ contaminated diet showed a significant increase in TSH and a significant decrease in $\mathrm{T}_{3}$ $\& \mathrm{~T}_{4}$ compared to control group. The significant decrease in $T_{3}$ and $T_{4}$ has been shown by Salem \& Selim (1994), Eraslan et al. (2005) and Hassan et al. (2010) which was in agreement with these results. The levels of blood $\mathrm{T}_{3}$ and $\mathrm{T}_{4}$ in all treated groups $\left(\mathrm{G}_{2}-\mathrm{G}_{5}\right)$ were decreased compared to $\left(G_{1}\right)$. The mechanism concerning the decrease in serum $\mathrm{T}_{3}$ and $\mathrm{T}_{4}$ levels may be associated with the decrease in blood iodine level, which is essentially significant in the synthesis of these hormones (Markou et al., 2001). It is obvious that AFs residues cause damage of the epithelium cells of the digestive tract (Johri et al., 1990). The $\mathrm{T}_{3} \& \mathrm{~T}_{4}$ in the groups $\left(\mathrm{G}_{3}, \mathrm{G}_{4} \& \mathrm{G}_{5}\right)$ received the prepared hen's meal of aflatoxicated and $\gamma$-irradiated ration, were tended to reach the values as in the control group. As for the sexual hormones (estradiol, testosterone and progesterone) a significant decrease is clearly shown in group of non-irradiated AFs diet $\left(\mathrm{G}_{2}\right)$. These results are in concomitant with those reported by EL-Shewy \& Ebrahem (2004), Hasanzadeh et al. (2011) and Adedara et al. (2014). These authors also reported that Aflatoxins are very potent toxins affecting the growth of all animals, delay in genital system growth (Hafez et al., 1982), high disturbances in estrous cycle, reduced pregnancy rate and number of live new born, failure indication and intrauterine death of the foetus (Shapour \& Saeedeh, 2013). In this study, the results were confirmed by Kourousekos et al. (2008) who also proposed that $\mathrm{AFB}_{1}$ has a direct effect either on ovarian secreting cells or on the hypothalamus-hypophysis-ovarian axis. Most authors' hypotheses focus on the fact that AFs may affect the reproductive system by its toxic effect on the liver, where the cellular hepatic damage could inhibit enzyme synthesis and/or enzyme activity, inhibition of lipid metabolism or fatty acid synthesis, which is essential for synthesis of precursor molecules for AFs may influence the reproductive system by its toxic effect on the liver, where the cellular hepatic damage could inhibit enzyme synthesis and/or enzyme activity, inhibition of lipid metabolism or fatty acid synthesis, causing decreased synthesis of precursor molecules of gonadal as well as gonadotropic hormones (Handan \& Guleray, 2005).

The improvement in thyroid and sexual hormones of $G_{3}, G_{4}$ and $G_{5}$ may be due to effectiveness of $\gamma$-radiation in degradation of $\mathrm{AFB}_{1}$ for those hens fed irradiated contaminated diets that leads to decrease of aflatoxin residue in prepared hens' meals. An improvement was noticed also in a recovery period through keeping the same trend as experimental duration.

The amelioration in most tested parameters in both experimental and recovery periods are radiation dose dependant. The $30 \mathrm{kGy}$ dose is safe and effective for modulation of $\mathrm{AFB}_{1}$ deleterious effects on poultry production and could protect consumers health. 
TABLE 5. Creatinine, serum proteins of the experimental rats in different treated groups (T) and during experimental duration $(D)$ and recovery duration $(R)$.

\begin{tabular}{|c|c|c|c|c|c|c|}
\hline $\begin{array}{l}\text { Duration } \\
\text { (week) }\end{array}$ & & $\begin{array}{l}\text { Experimental } \\
\text { treated groups } \\
\text { (T) }\end{array}$ & $\begin{array}{c}\text { Creatinine } \\
\left(\mathrm{mg.dl}^{-1}\right)\end{array}$ & $\begin{array}{l}\text { Total protein } \\
\left(\text { g.dl }^{-1}\right)\end{array}$ & $\begin{array}{l}\text { Albumin } \\
\left(\text { g.dl }^{-1}\right)\end{array}$ & Globulin $\left(\mathrm{g.dl}^{-1}\right)$ \\
\hline \multirow{15}{*}{$\begin{array}{l}\text { Experimental } \\
\text { duration } \\
\text { (D) }\end{array}$} & \multirow{5}{*}{$1^{\text {st }}$} & $\mathrm{G}_{1}$ & $1.37^{\mathrm{d}} \pm 0.01$ & $7.73^{\mathrm{a}} \pm 0.06$ & $4.30^{\mathrm{a}} \pm 0.12$ & $3.43^{\mathrm{a}} \pm 0.09$ \\
\hline & & $\mathrm{G}_{2}$ & $1.71^{\mathrm{a}} \pm 0.01$ & $4.70^{\mathrm{e}} \pm 0.09$ & $2.47^{\mathrm{e}} \pm 0.05$ & $2.22^{c} \pm 0.06$ \\
\hline & & $\mathrm{G}_{3}$ & $1.62^{\mathrm{b}} \pm 0.02$ & $5.44^{\mathrm{d}} \pm 0.03$ & $2.96^{\mathrm{d}} \pm 0.02$ & $2.48^{\mathrm{b}} \pm 0.01$ \\
\hline & & $\mathrm{G}_{4}$ & $1.58^{\mathrm{b}} \pm 0.01$ & $5.94^{\mathrm{c}} \pm 0.04$ & $3.27^{\mathrm{c}} \pm 0.09$ & $2.67^{b} \pm 0.07$ \\
\hline & & $\mathrm{G}_{5}$ & $1.49^{c} \pm 0.01$ & $6.60^{\mathrm{b}} \pm 0.08$ & $3.91^{\mathrm{b}} \pm 0.05$ & $2.69^{\mathrm{b}} \pm 0.10$ \\
\hline & \multirow{5}{*}{$2^{\text {nd }}$} & $\mathrm{G}_{1}$ & $1.35^{\mathrm{e}} \pm 0.01$ & $7.86^{\mathrm{a}} \pm 0.06$ & $4.34^{\mathrm{a}} \pm 0.11$ & $3.52^{\mathrm{a}} \pm 0.15$ \\
\hline & & $\mathrm{G}_{2}$ & $1.78^{\mathrm{a}} \pm 0.02$ & $4.51^{\mathrm{e}} \pm 0.06$ & $2.31^{\mathrm{e}} \pm 0.02$ & $2.80^{b} \pm 0.05$ \\
\hline & & $\mathrm{G}_{3}$ & $1.57^{\mathrm{b}} \pm 0.02$ & $5.30^{\mathrm{d}} \pm 0.02$ & $2.85^{\mathrm{d}} \pm 0.01$ & $2.63 b^{c} \pm 0.04$ \\
\hline & & $\mathrm{G}_{4}$ & $1.50^{\mathrm{c}} \pm 0.02$ & $5.77^{\mathrm{c}} \pm 0.02$ & $3.14^{\mathrm{c}} \pm 0.02$ & $2.45^{\mathrm{cd}} \pm 0.01$ \\
\hline & & $\mathrm{G}_{5}$ & $1.41^{\mathrm{d}} \pm 0.01$ & $6.40^{\mathrm{b}} \pm 0.09$ & $3.59^{\mathrm{b}} \pm 0.04$ & $2.20^{\mathrm{d}} \pm 0.08$ \\
\hline & \multirow{5}{*}{$3^{\text {rd }}$} & $\mathrm{G}_{1}$ & $1.36^{\mathrm{d}} \pm 0.02$ & $7.74^{\mathrm{a}} \pm 0.04$ & $4.31^{\mathrm{a}} \pm 0.10$ & $3.43^{\mathrm{a}} \pm 0.07$ \\
\hline & & $\mathrm{G}_{2}$ & $1.87^{\mathrm{a}} \pm 0.02$ & $4.40^{\mathrm{e}} \pm 0.07$ & $2.25^{\mathrm{e}} \pm 0.02$ & $2.14^{\mathrm{d}} \pm 0.05$ \\
\hline & & $\mathrm{G}_{3}$ & $1.52^{\mathrm{b}} \pm 0.01$ & $5.0^{\mathrm{d}} \pm 0.07$ & $2.68^{b} \pm 0.01$ & $2.39^{c} \pm 0.06$ \\
\hline & & $\mathrm{G}_{4}$ & $1.43^{c} \pm 0.01$ & $5.54^{c} \pm 0.03$ & $2.94^{\mathrm{c}} \pm 0.02$ & $2.60^{\mathrm{b}} \pm 0.05$ \\
\hline & & $\mathrm{G}_{5}$ & $1.37^{\mathrm{cd}} \pm 0.01$ & $6.00^{b} \pm 0.10$ & $3.29^{\mathrm{b}} \pm 0.04$ & $2.70^{b} \pm 0.05$ \\
\hline \multirow{15}{*}{$\begin{array}{l}\text { Recovery } \\
\text { duration } \\
\text { (R) }\end{array}$} & \multirow{5}{*}{$4^{\text {th }}$} & \multirow{15}{*}{$\begin{array}{l}\text { Commercial non- } \\
\text { contaminated } \\
\text { diet with AFB1 }\end{array}$} & $1.33^{\mathrm{d}} \pm 0.01$ & $7.64^{\mathrm{a}} \pm 0.18$ & $4.39^{\mathrm{a}} \pm 0.09$ & $3.25^{\mathrm{a}} \pm 0.28$ \\
\hline & & & $1.81^{\mathrm{a}} \pm 0.03$ & $4.91^{\mathrm{d}} \pm 0.08$ & $2.47^{\mathrm{e}} \pm 0.04$ & $2.44^{\mathrm{b}} \pm 0.06$ \\
\hline & & & $1.50^{\mathrm{b}} \pm 0.01$ & $5.44^{\mathrm{c}} \pm 0.06$ & $3.01^{\mathrm{d}} \pm 0.02$ & $2.42^{\mathrm{b}} \pm 0.04$ \\
\hline & & & $1.40^{c} \pm 0.01$ & $6.03^{b} \pm 0.08$ & $3.32^{\mathrm{c}} \pm 0.02$ & $2.71^{\mathrm{b}} \pm 0.08$ \\
\hline & & & $1.36^{\mathrm{cd}} \pm 0.01$ & $6.34^{\mathrm{b}} \pm 0.04$ & $3.65^{\mathrm{b}} \pm 0.07$ & $2.68^{\mathrm{b}} \pm 0.08$ \\
\hline & \multirow{5}{*}{$5^{\text {th }}$} & & $1.34^{\mathrm{c}} \pm 0.01$ & $7.73^{\mathrm{a}} \pm 0.04$ & $4.36^{\mathrm{a}} \pm 0.05$ & $3.37^{\mathrm{a}} \pm 0.09$ \\
\hline & & & $1.75^{\mathrm{a}} \pm 0.02$ & $4.95^{\mathrm{e}} \pm 0.08$ & $2.62^{\mathrm{e}} \pm 0.05$ & $2.33^{\mathrm{c}} \pm 0.11$ \\
\hline & & & $1.46^{\mathrm{b}} \pm 0.01$ & $5.56^{\mathrm{d}} \pm 0.02$ & $3.21^{\mathrm{d}} \pm 0.03$ & $2.35^{\mathrm{c}} \pm 0.02$ \\
\hline & & & $1.38^{\mathrm{c}} \pm 0.01$ & $6.12^{\mathrm{c}} \pm 0.02$ & $3.48^{\mathrm{c}} \pm 0.02$ & $2.63^{b} \pm 0.03$ \\
\hline & & & $1.33^{\mathrm{c}} \pm 0.01$ & $6.43^{\mathrm{b}} \pm 0.05$ & $3.71^{\mathrm{b}} \pm 0.04$ & $2.71^{\mathrm{b}} \pm 0.08$ \\
\hline & \multirow{5}{*}{$6^{\text {th }}$} & & $1.32^{\mathrm{c}} \pm 0.01$ & $7.80^{\mathrm{a}} \pm 0.09$ & $4.42^{\mathrm{a}} \pm 0.05$ & $3.38^{\mathrm{a}} \pm 0.14$ \\
\hline & & & $1.69^{\mathrm{a}} \pm 0.01$ & $5.23^{\mathrm{e}} \pm 0.07$ & $2.81^{\mathrm{e}} \pm 0.05$ & $2.42^{\mathrm{c}} \pm 0.08$ \\
\hline & & & $1.42^{\mathrm{b}} \pm 0.01$ & $5.78^{\mathrm{d}} \pm 0.01$ & $3.36^{\mathrm{d}} \pm 0.02$ & $2.41^{\mathrm{c}} \pm 0.02$ \\
\hline & & & $1.35^{\mathrm{c}} \pm 0.01$ & $6.38^{c} \pm 0.02$ & $3.69^{\mathrm{c}} \pm 0.02$ & $2.68^{\mathrm{b}} \pm 0.03$ \\
\hline & & & $1.32^{\mathrm{c}} \pm 0.01$ & $6.88^{\mathrm{b}} \pm 0.04$ & $4.01^{\mathrm{b}} \pm 0.06$ & $2.87^{b} \pm 0.06$ \\
\hline
\end{tabular}

Means of each value in the same column within each duration with the same letter are not significantly different $(\mathrm{P}<0.05)$. Each value represents mean \pm standard error. The $\mathrm{P}$ value of Duration (D), Treatment (T), D*T, Recovery (R), Treatment ( $\mathrm{T}), \mathrm{R}^{*} \mathrm{~T}$ was 0.0001 . 
TABLE 6. Serum blood of TSH, $T_{3}, T_{4}$, estradiol, testosterone and progesterone of the experimental rats in different treated groups $(T)$ and during experimental duration (D) and recovery duration (R).

\begin{tabular}{|c|c|c|c|c|c|c|c|c|}
\hline & $\begin{array}{c}\text { Duration } \\
\text { (week) }\end{array}$ & $\begin{array}{l}\text { Experimental } \\
\text { treated } \\
\text { groups } \\
(\mathrm{T})\end{array}$ & $\begin{array}{c}\text { TSH } \\
(\mu \mathrm{IU} / \mathrm{ml})\end{array}$ & $\begin{array}{c}\text { T3 } \\
(\mathrm{ng} / \mathrm{ml})\end{array}$ & $\begin{array}{c}\text { T4 } \\
(\mu \mathrm{g} / \mathrm{dl})\end{array}$ & $\begin{array}{c}\text { Estradiol } \\
(\mathrm{pg} / \mathrm{ml})\end{array}$ & $\begin{array}{c}\text { Testosterone } \\
\text { (ng/ml) }\end{array}$ & $\begin{array}{c}\text { Progesterone } \\
\text { (ng/ml) }\end{array}$ \\
\hline \multirow{5}{*}{$\begin{array}{l}\text { Experimental } \\
\text { duration } \\
\text { (D) }\end{array}$} & \multirow{5}{*}{$3^{\text {rd }}$} & $\mathrm{G}_{1}$ & $0.17^{\mathrm{e}} \pm 0.01$ & $3.49^{\mathrm{a}} \pm 0.11$ & $21.54^{\mathrm{a}} \pm 0.45$ & $478.00^{\mathrm{a}} \pm 2.88$ & $2.40^{\mathrm{a}} \pm 0.11$ & $1.33^{\mathrm{a}} \pm 0.02$ \\
\hline & & $\mathrm{G}_{2}$ & $0.92^{\mathrm{a}} \pm 0.03$ & $1.73^{\mathrm{e}} \pm 0.11$ & $13.53^{\mathrm{e}} \pm 0.46$ & $239.66^{\mathrm{e}} \pm 4.37$ & $0.41^{\mathrm{e}} \pm 0.02$ & $0.57^{\mathrm{e}} \pm 0.03$ \\
\hline & & $\mathrm{G}_{3}$ & $0.55^{\mathrm{b}} \pm 0.01$ & $2.28^{\mathrm{d}} \pm 0.02$ & $15.82^{\mathrm{d}} \pm 0.34$ & $290.66^{\mathrm{d}} \pm 3.75$ & $0.92^{\mathrm{d}} \pm 0.02$ & $0.96^{\mathrm{d}} \pm 0.01$ \\
\hline & & $\mathrm{G}_{4}$ & $0.41^{\mathrm{c}} \pm 0.02$ & $2.54^{c} \pm 0.05$ & $17.46^{\mathrm{c}} \pm 0.16$ & $343.66^{c} \pm 6.88$ & $1.30^{c} \pm 0.05$ & $1.07^{c} \pm 0.03$ \\
\hline & & $\mathrm{G}_{5}$ & $0.30^{\mathrm{d}} \pm 0.02$ & $3.11^{\mathrm{b}} \pm 0.01$ & $19.23^{\mathrm{b}} \pm 0.05$ & $429.33^{b} \pm 3.17$ & $1.86^{\mathrm{b}} \pm 0.03$ & $1.22^{\mathrm{b}} \pm 0.02$ \\
\hline \multirow{5}{*}{$\begin{array}{l}\text { Recovery } \\
\text { duration } \\
\text { (R) }\end{array}$} & \multirow{5}{*}{$6^{\text {th }}$} & \multirow{5}{*}{$\begin{array}{l}\text { Commercial } \\
\text { non } \\
\text { contaminated } \\
\text { diet with } \\
\text { AFB1 }\end{array}$} & $0.33^{\mathrm{d}} \pm 0.02$ & $3.68^{\mathrm{a}} \pm 0.04$ & $24.01^{\mathrm{a}} \pm 0.62$ & $480.00^{\mathrm{a}} \pm 0.57$ & $2.47^{\mathrm{a}} \pm 0.07$ & $1.34^{\mathrm{a}} \pm 0.01$ \\
\hline & & & $0.95^{\mathrm{a}} \pm 0.01$ & $2.33^{\mathrm{e}} \pm 0.03$ & $17.01^{\mathrm{d}} \pm 0.41$ & $281.67^{\mathrm{d}} \pm 2.33$ & $0.93^{\mathrm{d}} \pm 0.03$ & $0.72^{\mathrm{e}} \pm 0.02$ \\
\hline & & & $0.90^{\mathrm{a}} \pm 0.02$ & $2.82^{\mathrm{d}} \pm 0.03$ & $19.50^{c} \pm 0.27$ & $334.00^{c} \pm 6.80$ & $1.27^{\mathrm{c}} \pm 0.02$ & $1.0^{\mathrm{d}} \pm 0.01$ \\
\hline & & & $0.68^{b} \pm 0.01$ & $3.21^{\mathrm{c}} \pm 0.02$ & $21.50^{\mathrm{b}} \pm 0.07$ & $391.33^{b} \pm 1.45$ & $1.76^{\mathrm{b}} \pm 0.03$ & $1.19^{\mathrm{c}} \pm 0.01$ \\
\hline & & & $0.53^{\mathrm{c}} \pm 0.02$ & $3.57^{b} \pm 0.02$ & $23.53^{\mathrm{a}} \pm 0.39$ & $470.67^{a} \pm 3.52$ & $2.44^{\mathrm{a}} \pm 0.02$ & $1.30^{\mathrm{b}} \pm 0.01$ \\
\hline
\end{tabular}

Means of each value in the same column within each duration with the same letter are not significantly different $(\mathrm{P}<0.05)$. Each value represents mean \pm standard error. The P value of Duration (D), Treatment (T), D*T, Recovery(R), Treatment (T), R*T was 0.0001

\section{Conclusion}

$\mathrm{AFB}_{1}$ is a major toxic contaminant of foods and feeds. The results of the present study suggest that treatment of feed with $\gamma$-irradiation at up $30 \mathrm{kGy}$ is safe and helpful to reduce the toxicity of $\mathrm{AFB}_{1}$ as well as improving the feed intake, live body weight, internal organ weights and blood biochemical attributes in the rats without any negative changes. These findings suggest that $\gamma$-irradiation treatment seemed to be successfully used safely to prevent any adverse effects of $\mathrm{AFB}_{1}$ at concentration level $0.2 \mathrm{mg} /$ $\mathrm{kg}$ in contaminated feed of lying and subsequently, produced safe animal products for consumers.

\section{References}

Adedara, I.A., Nanjappa, M.K., Farombi, E.O. and Akingbemi, B.T. (2014) Aflatoxin $\mathrm{B}_{1}$ disrupts the androgen biosynthetic pathway in rat Leydig cells. Food Chem Toxicol. 65, 252-259.

Abu-Aita, Nashwa A., Mansour, Mogda K., Moneir, Samer M. and Nada A.A. (2008) Influence of the probiotic pediococcus acidilactici on chromosomal aberrations, clininicopathological alterations and immunological changes in aflatoxicated rabbits. Vet. Med. J. Giza, 56.
Agag, M. (2004) Mycotoxins in foods and feeds 1-aflatoxins. Ass. Univ. Bull. Environ. Res. 7(1).

AOAC (1995) Aflatoxins in corn and peanut butter. In: "Official Methods of Analysis of AOAC International", Cunniff, P (Ed.), Vol. 2., AOAC International Gaithersburg, MD (Method 990, 33), 18.

Bhat, R., Ravishankar V.R. and Karim, A.A. (2010) Mycotoxins in food and feed: Present status and future concerns. Comprehensive Reviews in Food Science and Food Safety, (9), 57-81.

Bailey, C.A., Latimer, G.W., Barr, A.C., Wigle, W.L., Haq, A.U., Balthrop, J.E. and Kubena, L.F. (2006) Efficacy of montmorillonite clay (NovaSil PLUS) for protecting full-term broilers from aflatoxicosis. $J$. Appl. Poult. Res. 15, 198-206.

Bennett, J.W. and Klich, M. (2003) Mycotoxins. Clinical Microbiological Review, 16(3), 497-516.

Bintvihok, A., Thiengnin, S., Doi, L. and Kamagai, S. (2002) Residues of aflatoxin in liver, muscles and eggs of domestic Fowls. J. Vet. Sci. 6, 1037-1039.

Bryden, W.L. (2007) Mycotoxins in the food chain: Human health implications. Asia. Pac. J. Clin Nutr. 16(1), 95-101. 
Chibanga, J.F., Nyirenda, B.D. and Simbaya, J. (2014) Effect of supplementing different levels of vitamin $\mathrm{A}$ to aflatoxin $\mathrm{B}_{1}$ contaminated diets on the performance of broiler chickens. Asian J. Natur. Appl. Sci. 3(4), 35-45.

Coles, E.H. (1986) "Veterinary Clinical Pathology" $4^{\text {th }}$ ed., pp.10-79. W.B. Saunders Co., Philadelphia.

Denli, M., Blandon, J.C., Guynot, M.E., Salado, S. and Perez, J.F. (2009) Effects of dietary AflaDetox on performance, serum biochemistry, histopathological changes, and aflatoxin residues in broilers exposed to aflatoxin $\mathrm{B}_{1}$. Poult.Sci. 88, 1444-1451.

Duncan, D.B. (1955) Multiple range and multiple F tests. Biomet. 11, 1.

El-Ghany, W.A.A., Hatem, M.E. and Ismail, M. (2013) Evaluation of the efficacy of feed additives to counteract the toxic effects of aflatoxicosis in broiler chickens. Inter. J. Anim. Vet. Advan. 5, 171182.

El-Shewy, E. and Ebrahem, M.F. (2004) Ameliorative effect of vitamin $\mathrm{E}$ against the toxicity of aflatoxin $B_{1}$ on rats with special reference to its effect on male fertility, $1^{r s t}$ Ann. Confr. , FVM., Moshtohor, Sept, pp. 189-213.

EPA (Environmental Protection Agency) 402-F-12002. (2013) EPA's Radiation Protection Standards.

Eraslan, G., Essiz, D., Akdogan, M., Sahindokuyucu, F., Altintas, L. and Hismiogullari, S.E. (2005) Effects of dietary aflatoxin and sodium bentonite on some hormones in broiler chickens, Bull. Vet. Inst. Pulawy, 49, 93-96.

Farag, M., Diaa El-Din, H., Abdalla, E.A., Abdul Azeem, A.M and Ahmed, Nashwa A.H. (2017) The Use of radiation to suppress the impact of aflatoxin $\mathrm{B}_{1}$ contaminated diets on the productive performance and immunological response of laying hens. Arab J. Nucle. Sci. and Appli. 50(2), 162-180.

Farkas, J. (1989) Microbiological safety of irradiated foods - Review. Intl J. Food Microbiol. 9, 1-15.

Gaskill, C.L., Miller, L.M., Mattoon, J.S., Hoffmann, W.E., Burton, S.A., Gelens, H.C.J., Ihle, S.L., Miller, J.B., Shaw, D.H. and Cribb, A.E. (2005) Liver histopathology and liver serum alanine aminotransferase and akaline phosphatase activities in epileptic dogs receiving phenobarbital. Vet. Pathol. 42, 147-160.

Hafez, A.H., Megalla, S.E., Abdel-Fattah, H.M. and Kamel, Y.Y. (1982) Aflatoxin and aflatoxicosis. II. Effects of aflatoxin on ovaries and testicles in mature domestic fowl. Mycopathlogica, 77(3),137$139,9$.

Handan, U. and Guleray, A. (2005) Selenium protective activity against aflatoxin $\mathrm{B}_{1}$ adverse effects on Drosophila melanogaster. Braz. Arch. Biol. Technol. 48, 2.

Hasanzadeh, S., Hosseini, E. and Rezazadeh, L. (2011) Effects of aflatoxin $B_{1}$ on profiles of gonadotropic (FSH and LH), steroid (testosterone and $17 \beta$-estradiol) and prolactin hormones in adult male rat Iran. J. Vet. Res., Shiraz University, 12(4), 332-336.

Hassan, A.A., Rashid, M.A. and Koratum, K.M. (2010) Effect of aflatoxin $\mathrm{B}_{1}$, zearalenone and ochratoxin A on some hormones related to fertility in male rats. Life Sci. J. 7(3), 64-72.

Hussein, H.S. and Brasel, J.M. (2001) Toxicity, metabolism, and impact of mycotoxins on humans and animals. Toxicology, 167(2), 101-134.

IARC (2002) Some traditional herbal medicines, some mycotoxins, naphthalene, and styrene, IARC Monographs, 82, Lyon, IARC.

Ibeh, I.N. and Saxena, D.K. (1998) Effect of alphatocopherol supplementation on the impact of aflatoxin $\mathrm{B}_{1}$ on the testes of rats. Exp.Toxic.Pathol. 50, 221-224.

Johri, T.S., Sadagopan, V.R., Shrivastava, M.A. and Jumdar, S. (1990) Effect of dietary aflatoxin on the performance of purebred broiler chicks. Indian J. Anim. Sci. 60, 1246-1248.

Kabak, B., Dobson, A.D.W. and Var, I. (2006) Strategies to prevent mycotoxin contamination of food and animal feed: A review. Crit. Rev. Food Sci. Nutr. 46, 593-619.

Kourousekos, G.D., Theodosiadou, E., Ververidis, H., Belibasaki, S., Boscos, C., Saratsis, P. and Lymberopoulos, A.G. (2008) Effect of aflatoxin $\mathrm{B}_{1}$ administration on serum steroid hormones 
concentrations of goats. Reproduction in Domestic Animals, 43, 102.

Kramer, J.W. (1989) Clinical enzmology. In: "Clinical Biochemistry of Domestic Animals", (4 ${ }^{\text {th }}$ ed.), Kaneko, J.J. (Ed.), Academic Press, San Diego. 338.

Manegar, G. A., Shambulingappa, B. E. and Ananda,K. J. (2010) Studies on tolerance limitof aflatoxin in commercial broilers. Libyan Agri. Res. Cent. J. Intern. 1, 177-181.

Markou, K., Georgopoulos, N., Kyriazopoulou, V. and Vagenakis, A.G. (2001) Iodine-induced hypothyroidism. Thyroid, 11, 501-510.

Markov, K., Mihaljevic, B., Domijan, A.M., Pleadin, J., Delas, F. and Frece, J. (2015) Inactivation of aflatoxigenic fungi and the reduction of aflatoxin $\mathrm{B}_{1}$ in vitro and in situ using gamma radiation. Food Control, 54, 79-85.

Pasha, T.N., Farooq, M.U., Khattak, F.M., Jabbar, M.A. and Khan, A.D. (2007) Effectiveness of sodium bentonite and two commercial products as aflatoxin absorbents in diets for broiler chickens. Anim. Feed. Sci. Technol. 132, 103-110.

Perozo, F. and Rivera, S. (2003) Effect of aflatoxin B exposure and selenium supplementation on immune response in broilers. Indian Vet. J. 80, 1218-1221.

Salem, M.I. and Selim, M.(1994) Effect of aflatoxin $\mathrm{B}_{1}$ on thyroid hormones metabolism in young male albino rats. J. of Environmental Sciences, Mansoura Uinv. 7, 141-158.

SAS Institute Inc. (2004) SAS/ STAT 9.1 User's Guide. SAS Institute Inc., Cary, NC, USA.
Sharghi, Y. and Manafi, M. (2011) The additional effects of aflatoxin and T-2 toxin combination on commercial broilers: I: Effects on performance parameters and internal organs. Research J. Fisher. and Hydrobiol. 6, 549-553.

Shapour, H. and Saeedeh, A.(2013) Aflatoxin B effects on ovarian follicular growth and atresia in the rat. Comp.Clin. Pathol. 22, 563-572.

Shi, Y.H., Xu, Z.R., Feng, J.L. and Wang, C.Z. (2006) Efficacy of modified montmorillonite nanocomposite to reduce the toxicity of aflatoxin in broiler chicks. Anim. Feed Sci. Technol. 129, 138148.

Thaxton, J.P., Tung, H.T. and Hamilton, P.B. (1974) Immunosuppression In thrombocytes during aflatoxicosis. Poult. Sci. 58, 562-566.

Waje, C.K., Jun, S.Y., Lee, Y.K., Kim, B.N., Han, D.H., Jo, C. and Kwon, J.H. (2009) Microbial quality assessment and pathogen inactivation by electron beam and gamma irradiation of commercial seed sprouts. Food Contr. 20, 200-204.

Yosef, T.A., Al-Julaifi, M.Z., Salah-El-Dein, W.M. and AL-Rizqi, A.M. (2013) Assessment of aflatoxin M1 residues in raw cow milk at Al-Riyadh area with reference to some detoxification applications. Life Sci. J. 10, 3365-3369.

Zaghini, A., Martelli, G. and Roncada, P. (2005) Mannanoligosaccharides and Aflatoxin $\mathrm{B}_{1}$ in Feed for Laying Hens: Effects on Egg Quality, Aflatoxins $\mathrm{B}_{1}$ and $\mathrm{M}_{1}$ Residues in Eggs and Aflatoxin $\mathrm{B}$ Levels in Liver. Poult. Sci. 84, 825-832.

(Received 23/12/2017; accepted 7 / 2 /2018) 


\section{السلامه الصحية و الأمان السمي للحوم الاجاج البياض المغذي على علاثق ملوثه

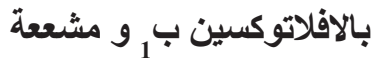

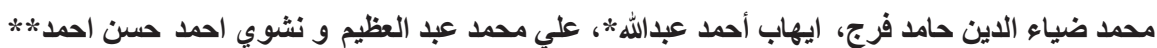

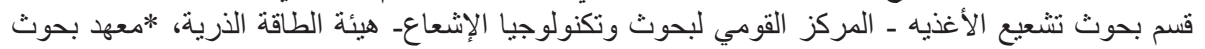

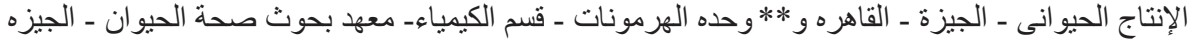

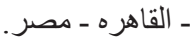

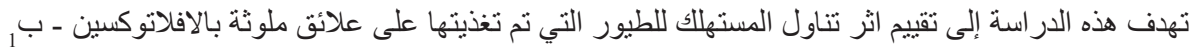

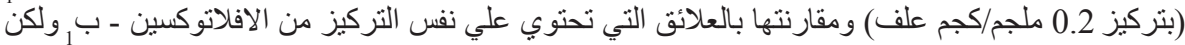

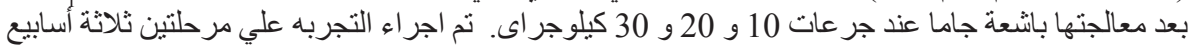

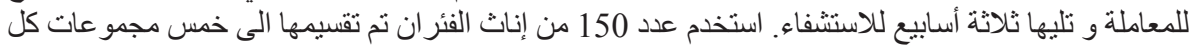

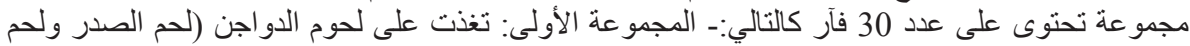

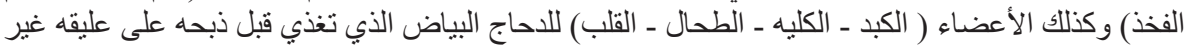

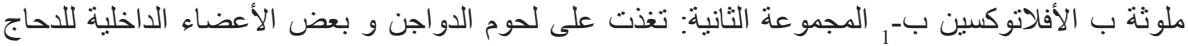

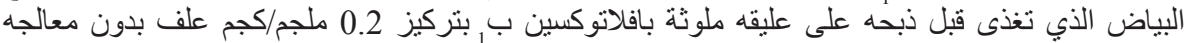

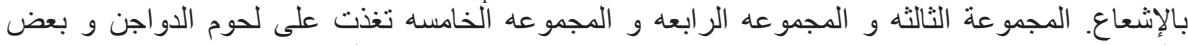

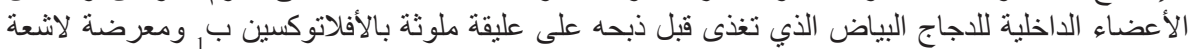

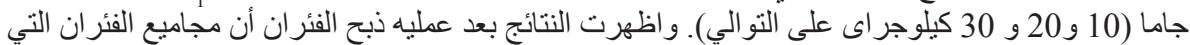

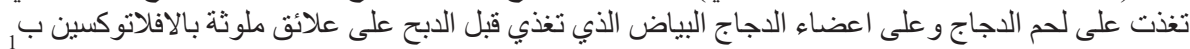

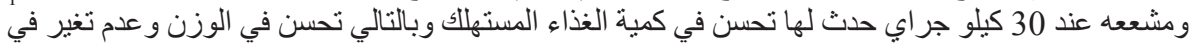

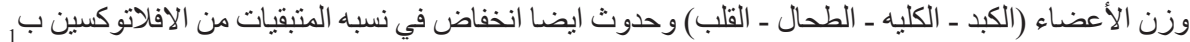

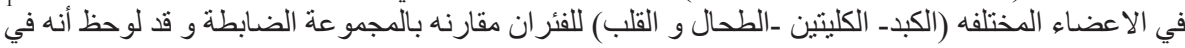

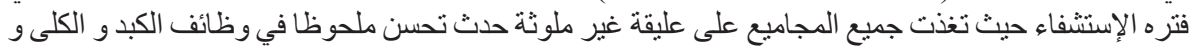

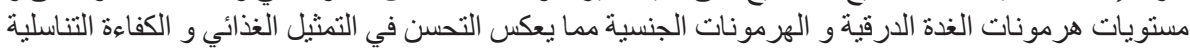

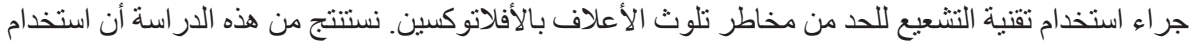

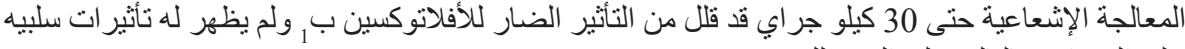

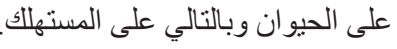

